Mots. Les langages du politique

Regards sur le post-colonialisme linguistique

\title{
Discours, normes et pouvoirs en hispanophonie. L'exemple de la promotion de l'espagnol au Brésil
}

Discourse, norms and seats of power in Spanish-speaking countries. The case of promoting Spanish in Brazil

Discurso, normas y poderes en hispanofonía. El ejemplo de la promoción del español en Brasil

\section{Virginia Garin}

\section{(2) OpenEdition}

\section{Journals}

Édition électronique

URL : https://journals.openedition.org/mots/21778

DOI : $10.4000 /$ mots. 21778

ISSN : 1960-6001

Éditeur

ENS Éditions

\section{Édition imprimée}

Date de publication : 31 décembre 2014

Pagination : 43-58

ISBN : 978-2-84788-544-6

ISSN : 0243-6450

\section{Référence électronique}

Virginia Garin, «Discours, normes et pouvoirs en hispanophonie. L'exemple de la promotion de

l'espagnol au Brésil », Mots. Les langages du politique [En ligne], 106 | 2014, mis en ligne le 31 décembre 2016, consulté le 23 avril 2022. URL : http://journals.openedition.org/mots/21778 ; DOI : https:// doi.org/10.4000/mots. 21778 


\section{Discours, normes et pouvoirs en hispanophonie. L'exemple de la promotion de l'espagnol au Brésil}

L'année 1492 marque le début d'une histoire commune entre l'Espagne et le continent latino-américain. La célèbre et prémonitoire phrase d'Antonio de Nebrija dans le Prólogo à sa Gramática Castellana parue la même année : «la langue a toujours été la compagne de l'Empire», donne le ton des relations qu'entretiendront pendant plus de cinq cents ans l'Espagne et ses anciennes colonies. Cette histoire commune marquée par les éloignements et les rapprochements, que ce soit au niveau politique, économique, culturel ou linguistique, se poursuit aujourd'hui dans le contexte de la mondialisation et du capitalisme tardif. On assiste depuis le début des années quatre-vingt-dix à une nouvelle ère des politiques linguistiques au sein du monde hispanique où l'on voit apparaître ce que Monica Heller, entre autres, appelle une commodification du langage (Heller, 2010). La langue espagnole devient dans les discours et dans les faits une marchandise que l'on peut négocier dans un marché donné. La rentabilité économique de la langue fait l'objet de projets de recherche subventionnés par des entreprises multinationales espagnoles et soutenus par les autorités de la langue ${ }^{1}$, on lui consacre des congrès ${ }^{2}$ et les médias, notamment la presse écrite, en font un sujet privilégié (Garin, 2014). Selon Del Valle et Villa (2007), l'exploitation du poids économique de l'espagnol est planifié autour de deux projets principaux : le développement de l'industrie linguistique d'enseignement et de diffusion de l'espagnol langue étrangère (désormais ELE) et l'augmentation de la consommation de produits culturels en langue espagnole (musique, cinéma, littérature, etc.).

1. Nous faisons allusion au projet de recherche intitulé «La valeur économique de l'espagnol: une entreprise multinationale », conduit par trois professeurs d'économie entre 2006 et 2012 et parrainé par la fondation Telefónica et le Secrétariat général Ibéro-américain (SEGIB) avec le soutien de l'Institut Cervantes et du Real Instituto Elcano.

2. La rentabilité de la langue est l'un des sujets récurrents des Congrès internationaux de la langue espagnole (CILE). Ce sont des forums de réflexion dans lesquels des spécialistes discutent de la situation de l'espagnol dans le monde. Ce sont des événements plus politiques que scientifiques organisés tous les trois ans, depuis 1997, dans une ville espagnole ou latino-américaine par l'Institut Cervantes, la RAE, l'Association des académies de la langue espagnole ainsi que par les gouvernements de l’Espagne et du pays qui accueille le congrès.

Université Montpellier III, DIPRALANG (EA 739)

garin.virginia@gmail.com

Mots. Les langages du politique $\mathrm{n}^{\circ} 106$ novembre $2014 \bullet 43$ 
C'est avec la création de l'Institut Cervantes en 1990 que l'Espagne se lance dans la compétition des langues «sur l'échiquier mondial» (Maurais, 2001 p.7). L'Institut Cervantes est une institution publique espagnole qui dépend du ministère des Affaires étrangères et du ministère de la Culture et avec pour président honoraire le roi d'Espagne en personne. Cette institution vient s'ajouter à d'autres agents de la politique linguistique et culturelle extérieure de l'Espagne comme l'Agence espagnole de coopération internationale pour le développement (AECID) ou l'organisme Action culturelle espagnole (ACE). Ce dispositif de politique linguistique et culturelle au sein de l'État espagnol est bien évidemment étroitement lié à la vie politique du pays. Cela se manifeste, par exemple, dans le fait que l'élection d'un nouveau gouvernement s'accompagne d'un grand renouvellement au sein des directions des centres Cervantes dans le monde. Les postes de directeur sont en effet des postes politiques qui, contrairement à d'autres postes de titulaires, ne font pas l'objet d'un concours.

L'Institut Cervantes est également épaulé, dans sa mission de diffusion de l'espagnol dans le monde, par l'institution responsable de veiller sur la langue, l'Académie royale espagnole (désormais RAE). Le nouveau directeur de l'Institut Cervantes et ancien directeur de la RAE, Víctor García de la Concha, rappelle dans son discours de prise de fonctions en 2012 la réaction de son prédécesseur, Fernando Lázaro Carreter, lors de la création de l'Institut Cervantes: «Nous, les académiciens, sommes les pères conciliaires et le Cervantes formera et enverra dans le monde entier des missionnaires pour qu'ils enseignent l'évangile l'espagnol. »3 Outre la référence évidente au passé colonial espagnol et l'idéologie qu'une telle allusion laisse entrevoir, ce commentaire décrit assez bien les liens étroits qui unissent les deux institutions en charge de la politique linguistique espagnole4. C'est ensemble que, depuis la fin des années quatrevingt-dix, la RAE et l'Institut Cervantes mènent une politique linguistique panhispanique, accompagnés par l'Association des académies de la langue espagnole (ASALE) réunissant toutes les académies sœurs de l'Académie espagnole depuis 1951. Comme on peut le lire sur le site internet de la RAE, cette politique linguistique implique une collaboration avec chacune des vingt-deux académies de la langue. Les œuvres qu'elles soutiennent (dictionnaire, grammaire et orthographe) sont le fruit d'une responsabilité commune dont l'objectif est

3. «Los Académicos somos los padres conciliares y el Cervantes formará y enviará a todo el mundo misioneros que enseñarán el evangelio del español. » Discours de Víctor García de la Concha, 1erfévrier 2012, disponible sur: http://www.cervantes.es/sobre_instituto_cervantes/ prensa/2012/noticias/nota-discurso-toma-posesion-victor-garcia-de-la-concha.htm. La validité des liens mentionnés dans cet article a été vérifiée en novembre 2013. Toutes les traductions présentées dans cet article ont été réalisées par nos soins.

4. Le recours au lexique du champ sémantique de la conquête ou de la guerre est fort récurrent dans les discours des autorités de la langue, mais aussi dans celui de la presse écrite espagnole, comme cela a été analysé dans Garin, 2014. 
de rendre compte de l'unité de la langue tout en respectant «sa riche variété »5. Avec cette politique panhispanique s'opère un changement au niveau discursif visant à transformer la vieille image de la RAE, une institution qui, depuis les origines, «nettoie, fixe et donne de la splendeur ${ }^{6}$ et qui aujourd'hui, veut avant tout défendre «l'unité dans la diversité». Du point de vue normatif, cela implique une ouverture des esprits de la part des membres de la RAE qui reconnaissent désormais ouvertement le caractère pluricentrique de la norme de l'espagnol : "L'espagnol, de par son caractère de langue supranationale, est en réalité un ensemble de normes diverses partageant cependant une large base commune » (ASALE et RAE, 2004, p. 9)7.

On peut affirmer qu'il existe aujourd'hui dans le domaine hispanique plusieurs normes régionales basées sur les usages linguistiques «cultivés» des locuteurs appartenant aux secteurs de la société jouissant d'un important capital culturel et économique. L'ensemble de ces normes cultivées forment ce que García de la Concha appelle l'espagnol total, c'est-à-dire que toutes les normes régionales reconnues de l'espagnol seraient sur un pied d'égalité et il n'y aurait guère une norme unique de l'espagnol, correspondant à celle de l'Académie de Madrid. Mais comme le rappelle Lara (2011), la publication en 2010 du dictionnaire d'américanismes (ASALE, 2010) vient confirmer le fait que pour l'Académie, il y a deux formes d'espagnol : l'espagnol péninsulaire que l'on trouve dans le Dictionnaire de la RAE (DRAE) - censé être un espagnol général - et celui des pays latino-américains, «sans admettre le fait qu'une langue ne peut pas être divisée en deux : l'espagnol est parlé intégralement dans chaque région du monde » (Lara, 2011, p. 336) ${ }^{8}$.

Selon Moreno Cabrera (2011), cette langue panhispanique continue à correspondre à la variante castillane connue sous le nom de péninsulaire centrale et qui serait, auxyeux de l'Académie, un élément essentiel pour le maintien de l'unité linguistique de l'espagnol et pour la légitimation du rôle de leader que joue la RAE dans le monde hispanique. Cette suprématie de l'institution espagnole et toute la politique linguistique expansionniste de l'Institut Cervantes seraient la preuve ou la confirmation de l'existence d'un impérialisme linguistique espagnol (Hamel, 2006 ; Del Valle, 2007; Mar-Molinero, Paffey, 2011).

L'une des façons les plus efficaces et significatives de promotion et de mise en évidence de l'espagnol en tant que grande langue dans le marché linguistique mondial est le résultat de la politique et de l'énergie investies par l'actuel et par les précédents gouvernements de Madrid. On pourrait bien sûr affirmer qu'il s'agit tout

5. Consultable sur le site http://www.rae.es.

6. Il s'agit de la désormais classique devise de la RAE : «limpia, fija y da esplendor».

7. «El español, porsu carácter de lengua supranacional, constituye en realidad un conjunto de normas diversas que, no obstante, comparten una amplia base común. "

8. " [...] sin reconocer el hecho de que una lengua no se puede partir en dos : el español se habla integralmente, en cada región del mundo hispánico [...]». 
simplement de la suite inévitable et prévisible de la domination impérialiste de l'Espagne au sein du monde hispanophone (Mar-Molinero, Paffey, 2011, p. 753).9

Dans cette contribution, notre objectif est d'analyser les politiques linguistiques en faveur de la diffusion de l'espagnol en nous intéressant particulièrement à la promotion de l'espagnol au Brésil. Le géant lusophone se présente aujourd'hui comme un marché incontournable pour les investissements espagnols et comme l'une des principales cibles de la politique linguistique espagnole ${ }^{10}$ dans un climat favorable à l'apprentissage de l'espagnol, motivé par la création en 1991 du Mercosur (Marché commun du sud). L'Institut Cervantes n'est pas passé à côté des possibilités d'expansion qui se présentaient à lui dans ce pays. Le Brésil est à l'heure actuelle le pays où l'Institut Cervantes compte le plus grand nombre de centres : huit au total, répartis dans les villes les plus importantes du pays. Cette présence n'a pas toujours été bien vécue par les responsables de la diffusion et de l'enseignement de l'espagnol localement - les enseignants et notamment les universitaires - qui ont parfois accusé l'Espagne, et plus particulièrement l'Institut Cervantes, de mener une politique néocolonialiste en Amérique latine.

La question de la norme linguistique de l'espagnol est aussi entrée dans le débat, réveillant en quelque sorte les vieilles tensions entre hispanistes et latino-américanistes. Nous avons souhaité nous pencher sur cet aspect de la diffusion de l'espagnol au Brésil au travers d'une étude des discours des professeurs des Instituts Cervantes du pays sur la politique linguistique panhispanique ainsi que sur la question de la norme à enseigner en contexte brésilien. Nos analyses s'appuient sur des entretiens semi-directifs réalisés entre le mois d'avril et de mai 2012 avec quarante-huit professeurs et coordinateurs pédagogiques de différentes nationalités dans sept Instituts Cervantes ${ }^{11}$.

9. "One of the most effective and significant ways that Spanish is being promoted around the world and made evident in the global linguistic market as a major language is the result of the policies and energy of current and recent Spanish governments in Madrid. It could of course be argued that this is simply an inevitable and to-be-expected continuation of imperial domination by Spain within the Spanish-speaking world. »

10. Les États-Unis sont l'autre grande cible de la politique linguistique extérieure espagnole. L'intérêt croissant pour l'espagnol dans ce pays ainsi que le nombre grandissant d'hispanophones (les prévisions affirment que ce sera le premier pays en nombre d'hispanophones en 2050) en font un marché incontournable pour l'industrie linguistique de l'espagnol.

11. Les entretiens ont été réalisés dans les Instituts Cervantes de Belo Horizonte, Brasilia, Porto Alegre, Recife, Rio de Janeiro, Salvador et Sao Paulo. La durée des entretiens oscille entre vingtcinq minutes et une heure quarante. La moitié des professeurs interrogés est de nationalité espagnole, ce qui en fait la nationalité la plus représentée dans notre échantillon. Cela s'explique par le fait que les professeurs effectifs, c'est-à-dire ceux qui ont passé un concours en Espagne, sont tous espagnols - à l'exception d'une enseignante argentine. Travaillant à temps plein à l'Institut Cervantes, ils étaient plus disponibles pour réaliser les entretiens que les professeurs recrutés sur place. 


\section{La diffusion de l’espagnol au Brésil}

Bien que l'espagnol ait longtemps été considéré au Brésil comme une langue de culture, son enseignement est resté marginal dans un système éducatif qui privilégiait d'autres langues de culture européennes comme l'anglais, le français ou l'italien. Cette situation commence à changer, principalement à partir de la création du Mercosur en 1991. Un autre facteur qui a suscité le regain d'intérêt pour l'espagnol et pour la culture hispanique au Brésil a été l'arrivée de nombreuses entreprises multinationales espagnoles, notamment dans les secteurs bancaire, énergétique et éditorial.

Le Mercosur, dont les États membres fondateurs sont l'Argentine, le Brésil, le Paraguay et l'Uruguay ${ }^{12}$, est une initiative transrégionale qui, bien qu'ayant vu le jour en tant que communauté économique, se présente aujourd'hui comme un projet d'intégration régionale également au niveau politique, social et culturel13.

Les questions linguistiques ont été évoquées dès la création du Mercosur. L'article 17 du traité d'Assomption stipule en effet que «Les langues officielles du Mercosur sont le portugais et l'espagnol »14. Le point no 4 du Protocole d’intentions de décembre 1991 mentionne «l'intérêt de promouvoir l'apprentissage des langues officielles du Mercosur - l'espagnol et le portugais - dans les systèmes éducatifs formels, non formels et informels ${ }^{15}$.

Jusqu'à présent, le pays qui s'est le plus sérieusement investi dans la promotion de l'apprentissage de la langue des États voisins sur son territoire est le Brésil. L'action la plus remarquable allant dans ce sens a été l'approbation de la loi $n^{0} 11.16 / 2005$ communément appelée «loi de l'espagnol » ${ }^{16}$ et qui a été un élément déterminant dans l'essor de l'espagnol au Brésil. Cette loi rend obligatoire l'offre d'apprentissage de la langue espagnole dans tous les établissements d'enseignement secondaire brésilien, créant ainsi un important marché potentiel pour les industries de la langue espagnole au Brésili7. Les pays membres du Mercosur ne disposant pas d'une infrastructure adaptée aux

12. Aujourd'hui, les États membres sont l'Argentine, le Brésil, le Paraguay, l'Uruguay, le Venezuela et la Bolivie qui est en cours d'adhésion. Les États associés sont le Chili, la Colombie, le Pérou et l'Équateur. L'admission de la Guyane et du Suriname en tant que membres associés du Mercosur est en cours de ratification.

13. La structure institutionnelle actuelle du Mercosur reflète assez bien ce projet d'intégration régionale à plusieurs niveaux. Disponible sur: http://www.mercosur.int/.

14. Le guarani a été déclaré langue officielle du Mercosur en 2006, mais les langues de travail restent pour l'instant le portugais et l'espagnol.

15. "El interés de difundir el aprendizaje de los idiomas oficiales del Mercosur - español y portugués - a través de los Sistemas Educativos; formales, no formales e informales. »

16. Approuvée par le président Luiz Inácio Lula Da Silva le 5 août 2005.

17. Pour une analyse plus détaillée du processus d'approbation de cette loi, voir Del Valle, Villa, 2007 ou Garin, 2013. 
nouveaux besoins linguistiques et éducatifs de leur voisin lusophone, l'Espagne a essayé de s'accaparer le marché brésilien grâce, principalement, au poids de l'industrie éditoriale espagnole au Brésil.

L'application de la loi rencontre cependant de multiples barrières, au nombre desquelles les effectifs insuffisants de professeurs formés en espagnol pour faire face assez rapidement à une demande aussi importante. L'Institut Cervantes, à propos duquel on a déjà vu l'intérêt manifeste qu'il portait pour le Brésil, essaiera d'apporter une solution à ce problème en signant un accord avec le Secrétariat d'éducation de l'État de São Paulo (SEE-SP), la banque Santander et le portail Universia ${ }^{18}$. L'objectif du projet nommé Oye était de former des enseignants (indépendamment de leur discipline de départ) ayant déjà un lien avec l'éducation nationale pour qu'ils deviennent des professeurs d'espagnol. Ils sont censés par la suite être habilités à donner des cours d'espagnol après avoir suivi une formation d'une charge horaire de 600 heures, entièrement à distance. Il est aisé d'imaginer à quel point cette résolution a pu être controversée si l'on considère que le volume horaire d'une licence d'espagnol à l'université brésilienne est de 2800 heures environ. Et c'est d'autant plus controversé que :

[...] avec ce projet, l'État déléguait à deux institutions étrangères (une banque et un organisme gouvernemental espagnol de diffusion de la langue) la fonction stratégique de former des enseignants du secondaire diminuant ainsi l'importance de l'expérience, des compétences et de toute la recherche développée dans les universités publiques qui, particulièrement dans l'État de São Paulo, ont été pionnières dans la formation des enseignants en la matière (Fanjul, 2010, p. 188).19

À la suite de la signature de cet accord, les associations de professeurs d'espagnol se sont mobilisées pour essayer d'empêcher l'entrée en vigueur du projet, manifestant ainsi leur mécontentement vis-à-vis du gouvernement - qui à leurs yeux dépréciait le travail et le rôle des universités - et vis-à-vis de l'Institut Cervantes, accusé de mener une politique mercantiliste et néocolonialiste en Amérique latine. Lors des entretiens que nous avons réalisés dans les centres Cervantes du Brésil, certains directeurs, responsables pédagogiques et professeurs ont fait mention de cette querelle. Ils condamnent, pour la plupart, l'attitude de certains universitaires qui, selon eux, cherchent à maintenir leur pouvoir et à rester les seuls référents de l'espagnol au Brésil, au grand détriment de la formation des professeurs et de l'application de la loi 11.161.

18. Il s’agit du plus grand réseau d’universités ibéro-américaines. Il a été créé en 2000 et bénéficie du parrainage de la banque Santander.

19. " [...] com esse projeto, o estado delegava a duas instituições estrangeiras (um banco e um órgão governamental espanhol de difusão da língua) a função estratégica de formar professores para atuarem no ensino médio, e desconsiderava a experiência, a competência e toda a pesquisa desenvolvida nas universidades públicas, que particularmente no estado de São Paulo, tinham sido pioneiras na formação de docentes na área. » 
Les nombreux débats qu'a suscités l'application de cette loi sont représentatifs des tensions qui existent à l'heure actuelle entre les agents de la politique linguistique espagnole, certaines associations de professeurs d'espagnol et de nombreux universitaires qui estiment que ce sont les pays membres du Mercosur qui devraient apporter leur soutien et leur aide au gouvernement brésilien pour l'application d'une loi née des accords signés au sein du bloc régional. Cette situation illustre assez bien les conflits qui peuvent apparaître dans le contexte actuel de la mondialisation où l'on a de plus en plus de mal à délimiter le champ d'action des États nationaux par rapport à celui des blocs régionaux, et où le marché et le jeu de l'offre et de la demande déterminent les décisions politiques y compris dans le domaine linguistique et culturel. Les tensions générées par la «loi de l'espagnol » révèlent la concurrence qui existe entre ceux qui définissent ce qui a de la valeur en tant que langue légitime et commercialisable et ceux qui contrôlent la production et la distribution des ressources linguistiques (Heller, 2010). Cela peut s'appliquer également à la question de la norme linguistique : quelle est la norme légitime? Quelle variante convient-il de commercialiser? Quelle norme choisissent ceux qui produisent et distribuent les ressources linguistiques?

\section{Le point de vue des professeurs de l'Institut Cervantes}

Même si l'Institut Cervantes reproduit le discours panhispanique de la RAE - qui reconnaît le caractère pluricentrique de la norme de l'espagnol - et se fait également écho du slogan d'unité dans la diversité, on peut observer que c'est la norme péninsulaire centrale, c'est-à-dire du centre-nord de l'Espagne, qui reste la norme privilégiée. Dans le Plan curricular de l'Institut Cervantes, un ouvrage servant de guide pédagogique pour l'enseignement de l'ELE et qui suit les consignes du Cadre européen commun de référence pour les langues, voici ce que l'on peut lire concernant le traitement de la norme linguistique et de la variation de l'espagnol:

L'extension géographique du monde hispanique et son grand nombre de locuteurs nous font prendre en considération sa complexité quand il s'agit de décrire la langue commune [...]. Ces présupposés ont été considérés lors de la sélection du matériel linguistique qui apparaît dans le Plan curricular [...] et qui correspond à la norme cultivée de la variété péninsulaire centrale espagnole. Le choix de cette variété s'appuie sur les traits communs qu'elle partage avec les autres normes cultivées du monde hispanique et sur sa projection au sein du modèle standardisé prestigieux propre à la communauté hispanique [c'est nous qui soulignons] (Plan curricular, 2007, p. 59). ${ }^{20}$

20. "La extensión geográfica de mundo hispánico y su elevado número de hablantes hacen necesario tener en cuenta su complejidad a la hora de describir la lengua común [...]. Esos presupuestos 
Selon Moreno Cabrera (2011), la politique linguistique panhispanique de la RAE et de l'Institut Cervantes cherche à faire passer une langue standard normative basée principalement sur une seule variante de l'espagnol d'Espagne - la variante péninsulaire centrale - pour une langue panhispanique qui serait la référence unique et correcte pour tout le monde hispanique. Dans l'extrait du Plan curricular ci-dessus, nous voyons clairement quels sont les arguments utilisés pour légitimer le choix normatif fait par l'Institut Cervantes. La norme choisie serait plus générale que les autres normes cultivées de l'espagnol (ce qui est fort discutable) et elle serait à la base du modèle standard prestigieux. Un autre argument mis en avant par le texte est le fait qu'il s'agit de la variante propre à l'institution chargée d'élaborer l'ouvrage, c'est-à-dire l'Institut Cervantes.

Les autres variantes de l'espagnol font l'objet d'une description que l'on pourrait qualifier d'informative et leur présentation n'est conseillée par le Plan curricular qu'à partir des niveaux supérieurs. On recommande aux professeurs de tirer parti, dans la mesure du possible, de la variété des accents et de l'origine des locuteurs d'espagnol pour démontrer la validité du modèle décrit et enseigné : «commun et neutre»(Plan curricular, 2007, p. 60). Les autres variantes de l'espagnol sont donc utilisées comme moyen de légitimation de la variante péninsulaire centrale en tant que modèle prestigieux unique parce qu'elle est la plus commune et la plus neutre de toutes.

Nous avons souhaité savoir si ces directives étaient appliquées et, dans l'affirmative, comment, dans le contexte brésilien où existe une grande diversité de nationalités parmi les professeurs de l'Institut Cervantes. Cette diversité découle de deux facteurs principaux : la proximité géographique des voisins hispanophones et les facilités juridiques de recrutement des travailleurs dans le cadre du Mercosur.

La prédominance de la variété péninsulaire centrale se fait notamment sentir dans les manuels scolaires utilisés ${ }^{21}$ et dans les instances d'évaluation proposées par l'institut, notamment dans les examens internationaux du DELE (Diplôme d'espagnol langue étrangère). Cela induit deux types principaux d'attitudes chez les professeurs interviewés. Il ya, d'une part, ceux qui privilégient leur propre variété en estimant que faire autrement serait trahir en quelque sorte leur identité propre. Cependant, ils reconnaissent qu'il existe une prédominance, certains parlent même d'une domination, de la variété péninsulaire centrale dans les manuels scolaires. D'autre part, il y a ceux qui semblent assu-

han sido considerados a la hora de seleccionar el material lingüistico que aparece en el Plan curricular [...] y que corresponde preferentemente a la variedad centro-norte peninsular española. La selección de esta variedad se sustenta en los rasgos comunes que comparte con las restantes normas cultas del mundo hispánico y en su proyección dentro del modelo estandarizado prestigioso de la lengua para la propia comunidad hispánica. "

21. On rappelle que les maisons d'édition espagnoles sont très puissantes au Brésil. Cela a des conséquences importantes sur le marché des livres didactiques en espagnol. 
mer avec moins de difficultés le fait qu'ils font partie d'une institution espagnole et trouvent normal que la variété péninsulaire soit la norme privilégiée. Voici des extraits d'entretiens illustrant ces tendances :

Moi j'enseigne ce que je sais, ce que je suis capable de parler naturellement [...] J'essaie toujours de leur présenter la variation de l'espagnol mais comme nous avons cette domination des livres didactiques d'Espagne avec la variété d’Espagne, elle est là, je l'enseigne mais je leur dis toujours qu'il y a d'autres structures, d'autres façons de dire; je leur montre ce que je sais, je leur parle de l'Uruguay, je ne vais pas leur parler d'endroits que je ne connais pas. Parfois apparaît du vocabulaire dans le livre et moi je leur donne une feuille à moi avec du vocabulaire pour compléter. C'est marrant parce qu'ils me demandent: «Madame, c'est quoi ordenador? » et je leur réponds : computadora. ${ }^{22}$

\section{Voici ce que dit par ailleurs une enseignante argentine :}

Quand je parle à mes étudiants, je parle dans ma variété porteña 23 et j'enseigne avec mon accent porteño mais la grammaire que j'enseigne c'est celle des livres, c'est celle qui utilise les pronoms tú et vosotros, c'est-à-dire l'espagnol d'Espagne parce que les livres sont espagnols, mais moi je ne modifie pas mon accent [...] ça me paraît très autoritaire de le faire [...] si on modifie sa façon de parler cela devient irréel, c’est irréel et la langue, la communication doit être quelque chose de réel.24

La coexistence de différentes variétés diatopiques au sein de la classe est souvent difficile à gérer pour les professeurs, notamment pour les moins expérimentés dans le domaine de l'ELE ou n'ayant pas suivi une formation spécifique en didactique des langues et des cultures ${ }^{25}$. Plusieurs professeurs affirment avoir une connaissance insuffisante de la variation diatopique de l'espagnol, y compris de la variété péninsulaire-centrale présente dans les manuels pour ce qui est des professeurs latino-américains.

Le troisième extrait représente à notre avis le deuxième type d'attitude. Il s'agit d'un entretien réalisé avec une enseignante brésilienne qui affirme parler

22. "Yo enseño lo que sé, lo que consigo hablar naturalmente [...] Yo trato siempre de presentarles la variación del español siempre que puedo pero como tenemos esa dominación de los libros didácticos de España entonces la variedad de España está a hí, la enseño pero pongo ese pero, hay también estas otras formas de decir, vocabulario, estructuras, muestro también lo que sé, les hablo de Uruguay no voy a hablar de otros lugares que no conozco ni nada, a veces le ponen vocabulario y yo les doy una hojita con la mía por ejemplo, después ellos me preguntan, es muy gracioso, "profe ¿qué es ordenador?”-computadora. » Enseignante uruguayenne.

23. Les Porteños sont les habitants de Buenos Aires.

24. "Mi variedad es porteña y yo cuando hablo con mis alumnos hablo como porteña y les enseño con mi acento porteño pero la gramática que yo enseño que es la de los libros es la que utiliza los pronombres de tú y de vosotros es decir es el español de España porque los libros son españoles pero no modifico mi acento [...] me parece muy autoritario modificarlo [...] si uno modifica su manera de habla es irreal, es algo irreal y la lengua, la comunicación tiene que ser algo real.»

25. Les coordinateurs pédagogiques que nous avons pu interviewer nous ont fait part de la difficulté souvent rencontrée pour embaucher des professeurs formés spécifiquement en ELE ou dans des disciplines connexes, notamment dans les Instituts Cervantes du nord-est du Brésil plus éloignés géographiquement des pays voisins hispanophones. 
un espagnol qu'elle qualifie de brésilien. Pour elle, la question de la norme et de la variété à enseigner n'est pas primordiale à partir du moment où les étudiants sont capables de communiquer en espagnol. Par rapport au traitement de la variation dans les manuels, elle dit que :

C'est un sujet un petit peu compliqué; moi je pense que si le matériel vient d'Espagne il est d'Espagne; c'est-à-dire que si l'Amérique latine veut être représentée, il faut qu'elle produise son propre matériel; je crois que les Espagnols font l'effort d'ajouter des choses [d'Amérique latine], mais je ne pense pas qu'ils soient obligés de le faire. ${ }^{26}$

Un autre extrait, cette fois-ci tiré d'un entretien réalisé avec un enseignant espagnol d'Andalousie, va dans le même sens :

Le Plan curricular de l'Institut Cervantes est un produit espagnol et il me paraît logique qu'il propose la variante espagnole, qui a entre autres l'avantage d'être une variante prestigieuse, même si cela reste discutable; bien sûr, pour moi elle peut l'être mais pas pour d'autres, mais il est vrai qu'il s'agit d'une variété très stable [...] c'est la variété la plus importante et géographiquement la plus puissante. 27

Dans ce dernier extrait, on observe comment le discours tenu dans le Plan curricular par rapport à la norme péninsulaire centrale coïncide avec le discours de ce professeur qui la considère comme étant la norme la plus digne d'être enseignée car elle est la plus importante et puissante de toutes ${ }^{28}$. Les professeurs sont parfois étonnés de ce qu'ils perçoivent comme une inaction de la part des pays membres du Mercosur en matière de diffusion linguistique de l'espagnol. Cette attitude justifierait le monopole exercé par l'Espagne dans ce domaine, notamment en ce qui concerne les manuels scolaires. Voici ce que dit à ce propos une enseignante espagnole :

- Pourquoi penses-tu qu'il n'y a pas de manuels didactiques faits ailleurs qu'en Espagne?29

- Je ne sais pas, ça me surprend parce que c'est curieux car parfois il y a des mouvements contre l'impérialisme espagnol, n'est-ce pas? Ce concept est toujours d'ac-

26. "Es un tema un poquito complicado yo pienso que si el material es de España es de España o sea que si Latinoamérica quiere ser representada tiene que producir materiales tiene que hacer sus propias cosas y yo creo que intentan bastante los españoles poner cosas [sobre América Latina] pero yo no siento que son obligados."

27. «El Plan curricular del Cervantes es un producto español y me parece lógico que proponga la variante española que tiene entre otras ventajas que es una variante que tiene prestigio aunque eso sea discutible claro tiene eso del prestigio para mi puede tenerlo para otros puede no tenerlo pero que es muy estable eso sí [...] es la variedad más importante digamos y geográficamente más potente."

28. La plupart des professeurs d'origine andalouse que nous avons pu interviewer semblent avoir une représentation plutôt négative de leur propre variété linguistique. Ils la considèrent moins claire et donc moins digne d’être enseignée que la variété péninsulaire centrale.

29. Il y en a, en réalité, mais très peu, et les professeurs affirment que souvent, ils ne sont pas aussi bons que les manuels espagnols. 
tualité, mais il n'y a pas beaucoup d'alternatives, c'est-à-dire, moi je pourrais tranquillement choisir un manuel fait en Argentine ou au Pérou [...] je vais peut-être avoir quelques problèmes mais c'est pareil pour mes collègues quand ils travaillent avec des livres espagnols et cela ne les empêche pas pour autant de faire leur travail, n'est-ce pas?30

Il est intéressant d'observer comment la dichotomie colonisé-colonisateur ou des allusions à l'impérialisme émergent dans le discours des professeurs. C'est le cas, par exemple, d'une enseignante uruguayenne qui nous explique le pourquoi de l'ouverture du discours actuel de la RAE et de sa nouvelle devise «unité dans la diversité»:

Cette politique a un rapport avec le contexte de mondialisation dans lequel on vit [...]. L’Espagne a besoin de cette union panhispanique, ibéro-américaine [...] ils ont [les Espagnols] besoin d'ouverture parce qu'aujourd'hui dans le monde sans frontières dans lequel on vit, le coup du pays bien hermétique, de la culture nationaliste qu'on emploierait comme si on vivait encore dans un contexte culturel colonial du siècle dernier, ça ne marche plus. Pour maintenir la marque espagnol [...] on a besoin de cette union ibéro-américaine ou panhispanique. Là on est ouverts à la diversité. ${ }^{1}$

La vision de cette enseignante nous semble bien fondée et il suffit de prêter attention aux discours actuels du directeur de l'Institut Cervantes, relevés notamment par la presse, pour comprendre qu'il y a une vraie volonté de rapprochement avec l'Amérique latine. La politique que mène actuellement García de la Concha au sein de l'Institut Cervantes rappelle en ce sens celle qu'il a menée pendant son mandat à la tête de la RAE : El Mundo le qualifiait ainsi, en février 2012, d' "homme qui a encouragé, depuis la RAE, le lien profond avec l’Amérique latine à travers la langue» (El Mundo, 1er février 2012)32.

En ce qui concerne la politique linguistique panhispanique menée depuis les années 2000 par la RAE, la plupart des professeurs interviewés se disent satisfaits de cette politique qui semble enfin donner la voix aux académies américaine et philippine. Même si la plupart y voient une réelle volonté de rapprochement entre la RAE et les autres académies - fruit d'une prise de conscience

30. "- ¿Por qué crees que no hay libros didácticos hechos fuera de España? - Pues no lo sé. Esto me llama la atención ¿no? Porque es curioso porque hay unas veces unas ondas de anti-imperialismo español ¿no ? Sigue vigente ese concepto pero no hay muchas alternativas es decir, yo podría tranquilamente elegir un libro hecho en Argentina o en Perú [...] claro que voy a tener a lo mejor algún problema, es como los que tienen mis compañeros cuando trabajan con libros españoles y eso no les impide desarrollar su trabajo ¿no?»

31. "Esta política tiene que ver con el contexto de globalización que vivimos hoy [...] España necesita de esta unión panhispánica o iberoamericana [...] necesitan de esta apertura en el contexto que vivimos de mundo sin fronteras, esto de nuestro país a hi cerradito, de nuestra cultura nacionalista y que vamos a emplearla como si fuera todavía el contexto cultural colonial del siglo anteriorya no funciona. Para mantener la marca español [...] es necesaria esa unión iberoamericana o panhispánica. Ahí estamos abiertos a la diversidad. »

32. "El hombre que impulsó desde la Real Academia de la Lengua el abrazo profundo con América Latina a través del idioma.» 
inévitable du poids des locuteurs hispanophones des Amériques ainsi que de leurs modalités linguistiques -, nombreux sont ceux qui se montrent plus critiques vis-à-vis de cette politique linguistique, n'y voyant qu'une politique tout court :

Je crois que cela [la politique linguistique panhispanique] est une question de politique, d'image et ça me fait de la peine que la langue devienne, en tout cas en ce qui concerne l'Espagne, un outil de promotion politique, économique et des entreprises [...] je pense que tout cela est aussi le reflet de ce prétendu panhispanisme.33

\section{Une Hispanophonie?}

Depuis le $19^{\mathrm{e}}$ siècle, à la suite de la naissance des nouvelles nations latino-américaines, l'Espagne s'efforce de développer une conscience communautaire partagée avec ses anciennes colonies (Del Valle, 2007). Le mouvement culturel connu sous le nom d'hispanisme ou d' «hispano-américanisme» reposait sur l'idée de l'existence d'une culture espagnole commune de part et d'autre de l'Atlantique. C'était pour l'Espagne une façon de garder des liens étroits avec ses anciennes colonies, mais toujours dans un désir de suprématie renforcé par la menace de l'impérialisme américain qui exerçait une influence de plus en plus forte sur le continent latino-américain. Néanmoins, la situation politique et économique de l'Espagne jusqu'à la fin des années soixante-dix limitait le champ d'action de ces efforts. Ce n'est que vers la fin des années quatre-vingt, après l'entrée de l'Espagne dans la Communauté économique européenne et à la consolidation de sa démocratie, que le pays est en mesure d'établir des liens politiques et économiques de nature différente avec les pays hispano-américains. Pendant les années quatre-vingt-dix, l’Espagne enregistre une croissance économique sans précédent grâce, entre autres, à l'internationalisation de son économie et à l'installation des entreprises espagnoles audelà des frontières du pays, notamment en Amérique latine. L'arrivée des capitaux espagnols sera qualifiée par certains, comme nous le rappelle Del Valle, d'une reconquête, cette fois-ci économique, du territoire latino-américain.

À chaque fois que j'allume la lumière, que je téléphone, que j'encaisse un chèque ou que je bois un verre d'eau, je suis en train de mettre de l'argent dans la poche de quelqu'un à Madrid... C'est comme si on était à nouveau une colonie et qu'on payait des impôts à la Couronne espagnole (le président du syndicat des employés de banque du Chili, Washington Post, 14 février 2000, cité par Del Valle, 2007, p. 55).34

33. "Yo creo que tiene un poco que ver con política, con imagen y es lo que me da un poco pena que la lengua se está convirtiendo ahora por lo menos desde el punto de vista de España en algo para promocionar política y económicamente y empresarialmente [...] yo creo que un poco todo eso es reflejo también de ese pretendido panhispanismo. » Enseignante espagnole.

34. "Cada vez que prendo la luz, llamo por teléfono, hago efectivo un cheque o tomo un vaso de 
Finalement, avec la création de la Communauté ibéro-américaine des nations (CIN) en 1991, dans le but de fortifier les relations entre les pays ibéroaméricains, l'Espagne trouve un espace politique adéquat lui permettant de garder des liens privilégiés avec l'Amérique latine et de renforcer le sentiment d'appartenance à une grande communauté internationale unie par une langue (avec le portugais) et une culture partagées35. Cette communauté ibéro-américaine, hispano-américaine ou panhispanique est rarement évoquée dans le domaine hispanique en tant qu'Hispanophonie. Del Valle (2007) utilise le terme hispanophonie pour faire allusion à une communauté imaginée, dans le sens d'Anderson (1983), forgée «sur la base d'une langue commune, également imaginée. Une langue commune qui unit, en créant un lien affectif, tous ceux qui croient la posséder et qui partagent un sentiment de loyauté envers celleci »36. Pour cet auteur, l'hispanophonie ne constitue donc pas seulement une communauté mais aussi une idéologie linguistique :

[...] celle-ci prend appui sur une vision de l'espagnol qui, d'une part, trouve son origine dans des institutions (la RAE et l'Institut Cervantes entre autres) dont les activités s'articulent autour de projets d'ordre économique (l'obtention d'un statut privilégié au sein du marché panhispanique) et politique (l'intégration des pays hispanophones en tant qu'entité politique internationale influente) et qui, d'autre part, naturalise et légitime ces projets en représentant la langue comme base d'une communauté unitaire et harmonieuse. (Del Valle, 2011, p. 568)37

En analysant les idées et les idéologies linguistiques qui émergent en ce début du $21^{\text {e }}$ siècle dans le domaine hispanique, Del Valle affirme que la langue est présentée actuellement aux hispanophones comme étant leur patrie

agua, estoy poniendo dinero en los bolsillos de alguien en Madrid... Es como si fuéramos una colonia de nuevo, pagando impuestos a la Corona española. "

35. Les discours cherchant à éveiller les sentiments d'appartenance communautaire dans le domaine hispanique trouvent leur équivalent dans les discours concernant la communauté ibéro-américaine. Dans un article écrit pour la revue Telos (2007) de la Fondation Telefónica, Enrique Iglesias, le secrétaire général du Secrétariat général ibero-américain (SEGIB) et ancien président de la Banque interaméricaine de développement (BID), déclarait : «La communauté ibéro-américaine des nations intégrée par les vingt-deux pays de langues espagnole et portugaise d’Amérique Latine et de la péninsule Ibérique, constitue un grand espace multinational fondé sur des principes et des valeurs ainsi que sur une identité culturelle et linguistique partagée [...] Pour cela, l'Ibéro-Amérique constitue probablement l'une des communautés de nations les plus naturelles, véritables et spontanées de la planète. Une communauté habituellement considérée comme "préexistante" à n'importe laquelle de ses formulations politiques et institutionnelles contemporaines.»

36. "[...] sobre la base de una lengua común - imaginada también -; una lengua común que une, formando un vínculo afectivo, a todos aquellos que se sienten en posesión de la misma y que comparten un sentimiento de lealtad hacia ella» (p. 37).

37. "La hispanofonía [...] se basa en una visión del español que, por un lado, emerge de instituciones (la RAE y el Cervantes entre otras) cuya actividad se coordina con proyectos de orden económico (la obtención de un estatus privilegiado en el mercado panhispánico) y político (la integración de los países hispanohablantes como entidad con peso político internacional) y, por otro, naturaliza y legitima estos proyectos al representar el idioma como base de una comunidad unitaria y armónica. » 
commune. La langue espagnole devient le lieu et le territoire où habitent tous les hispanophones du monde et auquel sont invités tous ceux qui veulent apprendre cette langue internationale et économiquement rentable. Une invitation qui a été principalement adressée à deux pays représentant de grands marchés linguistiques : les États-Unis et le Brésil.

L'Amérique latine est aujourd'hui une pièce maîtresse des projets d'expansion linguistique menés depuis l'Espagne. C'est grâce aux millions d'hispanophones répartis sur la géographie du continent américain que l'espagnol peut espérer se consolider en tant que langue globale et plus particulièrement grâce aux Latinos des États-Unis. La récente inauguration d'un observatoire de l'espagnol aux États-Unis, à la suite d'un accord signé entre l'Institut Cervantes et l'Université d'Harvard, est la preuve de l'intérêt porté à ce pays qui représente - tout comme le Brésil - un énorme marché économique pour les industries liées à l'espagnol.

Depuis un certain temps, l'on voit apparaître des discours qui mettent de plus en plus en valeur la dimension américaine de la langue (latino-américaine ou nord-américaine?). La situation économique actuelle de l'Espagne ne lui permettant pas de se lancer dans des projets d'expansion, comme ce fut le cas par le passé, il est devenu nécessaire de créer des liens plus solides avec les pays latino-américains pour s'assurer de la poursuite du projet expansionniste. $A B C$ soulignait ainsi en février 2012 que «García de la Concha souhaite que l'Institut Cervantes soit perçu en Amérique comme quelque chose

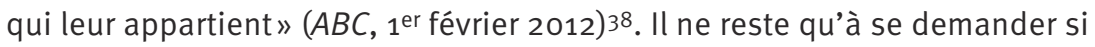
ce rapprochement se fera au profit, d'une manière ou d'une autre, des pays latino-américains, et si cette nouvelle vision américaniste ira jusqu'à changer les conceptions traditionnelles, toujours en vigueur quoique déguisées, de la norme légitime de l'espagnol à diffuser dans le monde au $21^{\mathrm{e}}$ siècle.

\section{Références}

Anderson Benedict, 1983, Imagined Communities. Reflections on the Origin and Spread of Nationalism, Londres, New York, Verso.

AsALE, RAE (Asociacion de academias de la lengua española y Real academia española), 2004, La nueva política lingüística panhispánica, RAE, Madrid.

Del Valle José, 2007, «La lengua, patria común. La hispanofonía y el nacionalismo panhispánico», J. Del Valle éd., La lengua ¿Patria común? Ideas e ideologías del español, Madrid, Iberoamericana, p.31-56.

- 2011, "Política del lenguaje y geopolítica. España, la RAE y la población latina de Estados Unidos», S. Senz, M. Alberte éd., El dardo en la Academia. Esencia y

38. "García de la Concha desea que todos los países de Hispanoamérica sientan al Instituto Cervantes como algo propio." 
vigencia de las academias de la lengua española, Santa Cruz de Tenerife, Melusina, t. I, p. 552-590.

Del Valle José, Villa Laura, 2007, «La lengua como recurso económico. Español S. A. y sus operaciones en Brasil», J. Del Valle éd., La lengua ¿Patria común ? Ideas e ideologías del español, Madrid, Iberoamericana, p. 97-127.

FAnjul Adrián Pablo, 2010, «São Paulo: o pior de todos. Quem ganha e o que se perde com a (não) introdução do espanhol na escola pública paulista», M.T. Celada, A. P. Fanjul, S. Nothstein éd., Lenguas en un espacio de integración. Acontecimientos, acciones, representaciones, Buenos Aires, Biblos, p. 185-204.

GARIN Viginia, 2013, "Quel espagnol enseigner au Brésil? Des politiques linguistiques aux représentations sociolinguistiques des acteurs didactiques», E. YasriLabrique, P. Gardies, K. Djordjevic Léonard éd., Didactique contrastive. Questionnements et applications, Actes du colloque de Béziers, 30 mai-1er juin 2011, Montpellier, Cladole, p.141-152.

- 2014, "La presse écrite espagnole et la mise en scène langagière des politiques linguistiques de diffusion de l'espagnol», K. Djordjevic, E. Yasri-Labrique éd., Médias et pluralisme, Paris, Archives contemporaines, p.189-215.

HAMEL Rainer Enrique, 2006, «The development of language empires», Sociolinguistics. An international Handbook of the Science of Language and Society, $2^{\mathrm{e}}$ édition, U. Ammon, N. Dittmar, K. Mattheier, P. Trudgill éd., Berlin, New York, De Gruyter, t. III, p. 2240-2258.

Heller Monica, 2010, "The commodification of language», The Annual Review of Anthropology, $\mathrm{n}^{0} 39$, p. 101-114.

IGLESIAS Enrique V., 2007, «El español en Iberoamérica. Lengua e identidad», Telos, $n^{\circ} 71$, en ligne : http://sociedadinformacion.fundacion.telefonica.com/telos/tribunagrabar.asp@idarticulo=1\&rev=71.htm (consulté le 4 juillet 2014).

InStituto Cervantes, 2007, Plan curricular del Instituto Cervantes. Niveles de referencia para el español, Madrid, Biblioteca Nueva, 3 tomes.

LARA Luis Fernando, 2011, «El símbolo, el poder y la lengua », S. Senz, M. Alberte éd., El dardo en la Academia. Esencia y vigencia de las academias de la lengua española, Santa Cruz de Tenerife, Melusina, t. I, p. 315-341.

Mar-Molinero Clare, Paffey Darren, 2011, «Linguistic imperialism. Who owns global Spanish?», M. Diaz-Campos éd., The Handbook of Spanish Sociolinguistics, Oxford, Blackwell, p.747-764.

MAURAIS Jacques, 2001, "Introduction. Vers un nouvel ordre linguistique mondial? », J. Maurais éd., Géostratégies des langues, Terminogramme, n99-100, automne, Québec, Les publications du Québec, p.7-33.

Moreno Cabrera Juan Carlos, 2011, "Unifica, limpia y fija”. La RAE y los mitos del nacionalismo lingüístico español», S. Senz, M. Alberte éd., El dardo en la Academia. Esencia y vigencia de las academias de la lengua española, Santa Cruz de Tenerife, Melusina, t. I, p.157-314. 
